J. Lake Sci. (湖泊科学), 2020, 32(1): 259-270

DOI 10. 18307/2020. 0124

(c) 2020 by Journal of Lake Sciences

\title{
柴达木盆地托素湖不同位置岩芯沉积物指标对比及其环境意义
}

\author{
丁宗艳, 张家武**,杨盼盼, 周 姗, 张玉枝 \\ （兰州大学资源环境学院,西部环境教育部重点实验室,兰州 730000)
}

\begin{abstract}
摘 要: 湖泊不同位置岩芯沉积物相同代用指标的变化是否一致对于重建可靠的区域气候变化历史至关重要. 目前多数 研究仅利用深水区单一的沉积岩芯来反演区域的气候环境变化, 对于不同位置岩芯重建结果的异同尚缺乏研究. 本文选 择托素湖不同位置、不同水深的 4 根短钻岩芯沉积物, 在放射性核素 $\left({ }^{210} \mathrm{~Pb}\right.$ 和 $\left.{ }^{137} \mathrm{Cs}\right)$ 定年的基础上, 对比分析各岩芯沉积 物粒度、碳酸盐含量等代用指标的变化情况. 结果显示, 湖泊内相近的沉积岩芯沉积物粒度变化相似, 但相距较远的岩芯 之间粒度变化差异较大; 浅水区的沉积物粒度 (粒径或组分含量) 在短时间尺度上变化很大, 而深水区的沉积环境比较稳 定, 在百年尺度上的变化不明显; 有机质与碳酸盐含量总体变化趋势一致且有较好的相关性, 表明碳酸盐可能受到湖泊 生产力的影响. 碳酸盐含量、碳酸盐氧同位素 $\left(\delta^{18} \mathrm{O}\right)$ 和 TL06 孔狍粉的 A/C 比值变化基本一致, 反映的有效湿度变化与同 期气象记录的相对湿度一致,但与降水量和蒸发量的变化不一致. 因此,托素湖粒度指标仅指示不同位置钻孔沉积环境 状况,与气候变化的关系并不明显,用碳酸盐及其同位素等地球化学指标恢复区域气候变化历史更可靠.
\end{abstract}

关键词: 平均粒径; 碳酸盐含量; 有机质; 代用指标; 托素湖; 柴达木盆地

\section{Comparison of sediment proxies of cores and their environmental significance at different locations of Lake Toson in Qaidam Basin*}

\section{DING Zongyan, ZHANG Jiawu ** , YANG Panpan, ZHOU Shan \& ZHANG Yuzhi}

( Key Laboratory of Western China's Environmental Systems, Ministry of Education, College of Earth Environmental Sciences, Lanzhou University, Lanzhou 730000, P.R.China)

\begin{abstract}
The consistency of proxies at different locations in a lake is of great importance to the reliability of regional climate reconstruction. At present, a single core in the central/deep lake was usually used to reconstruct the regional climate and environment change without verification. Here we compare the same proxy among the different core sediments from different locations of Lake Toson $\left(37^{\circ} 04^{\prime}-37^{\circ} 13^{\prime} \mathrm{N}, 96^{\circ} 50^{\prime}-97^{\circ} 03^{\prime} \mathrm{E}, 2808 \mathrm{~m}\right.$ a.s.l $)$, which is located in Delingha, NE Tibetan Plateau. Core TSL15S1, TSL15S3, TSL15S7 and TSLG17 were recovered at the water depth of 1, 16.5, 16.5 and $25 \mathrm{~m}$ in Lake Toson, respectively. Based on the dating of ${ }^{210} \mathrm{~Pb}$ and ${ }^{137} \mathrm{Cs}$ analyses, the changes of grain size, carbonate content and other proxies were compared and analyzed. The results show that the mean grain size is consistent at similar locations, while the grain size are quite different at distant locations within the lake. The grain size of sediments in the shallow water vary greatly in a short time scale, while the sedimentary environment in deep water is relatively stable, and the change is not obvious in the centurial. The variations of organic matter and carbonate content are consistent with each other, which indicates that carbonate may be affected by lake productivity. The organic matter content can reflect the size of lake productivity. The variations of carbonate content are consistent with the carbonate oxygen isotope in TSLC and the pollen A/C ratio of TL06, which was regarded as the substitute index of regional effective humidity. Therefore, the grain size in Lake Toson is reflecting the local sedimentary environment, and its relationship with the regional climate is not clear. Geochemical indexes are more reliable and are recommended for environment reconstructions in a well-mixed lake.
\end{abstract}

Keywords: Average particle size; carbonate content; organic matter; environmental proxies; Lake Toson; Qaidam Basin

* 2019-06-15 收稿;2019-08-15 收修改稿.

国家自然科学基金项目 (41771212) 和兰州大学中央高校基本科研业务费专项 ( lzujbky-2017-it81) 联合资助.

** 通信作者;E-mail: jwzhang@lzu.edu.cn. 
湖泊沉积具有覆盖面广、沉积连续的特点, 是进行高分辨率气候环境演化研究的重要载体 ${ }^{[1]}$. 以往利用 湖泊沉积物代用指标进行古气候环境重建的大多数研究均采用湖泊中单个沉积岩芯进行分析, 认为湖泊深 水区沉积物能较好地保存区域的环境变化信息 ${ }^{[2-9]}$. 但实际中多数湖泊的沉积物在空间上的分布并不均 匀 ${ }^{[10]}$, 尤其对于水文条件或湖底地形较为复杂的湖泊, 不同位置的沉积环境不同. 例如, 对青藏高原东北部 冬给措纳 ${ }^{[11]}$ 、哈拉湖 ${ }^{[12]}$ 不同位置的多个沉积岩芯对比发现, 湖泊中不同位置的沉积岩芯在粒度、碳酸盐含 量、 $\mathrm{C} / \mathrm{N}$ 等代用指标上均存在差异. 因此, 利用湖泊沉积物代用指标进行气候变化重建前, 应评估拟选取的 湖泊沉积物记录区域气候信号的潜力, 以避免将局地环境信息误认为是区域气候变化的可能.

青藏高原东北部的托素湖为典型的内陆封闭湖泊, 沉积物具有恢复区域气候环境变化的潜力 ${ }^{[13]}$. 但目 前该湖沉积记录研究较少, 不同位置沉积物记录气候环境信息的状况不清楚, 因此有必要开展湖泊近现代 以来沉积过程与区域气候环境变化关系的研究, 为进一步深人研究奠定基础. 本文用托素湖不同位置(不同 水深) 的 4 根短钻岩芯沉积物, 在精确定年的基础上, 分析各岩芯的粒度、有机质、碳酸盐含量及其同位素等 指标在湖泊内部的空间相似性和差异性, 与区域过去几十年气候环境变化的指标及器测资料对比分析, 评 估托素湖沉积物粒度、有机质和碳酸盐含量记录湖泊环境与区域气候信息状况, 为该湖及类似湖泊进行较 长时间尺度环境变化研究提供参考.

\section{1 研究区概况}

托素湖 ( $37^{\circ} 04^{\prime} \sim 37^{\circ} 13^{\prime} \mathrm{N}, 96^{\circ} 50^{\prime} \sim 97^{\circ} 03^{\prime} \mathrm{E}, 2808 \mathrm{~m}$ a.s.l) 位于青藏高原东北部柴达木盆地内, 距青海省 德令哈市约 $45 \mathrm{~km}$, 是巴音河流域的尾问湖泊 ${ }^{[14]}$ (图 1a). 托素湖面积 $145 \mathrm{~km}^{2[6]}$, 最大水深 $25 \mathrm{~m}$. 形态似三 角形, 在湖泊中心略偏北的位置有一大一小两个呈东南一西北走向的湖岛. 托素湖属咸水湖泊, 湖水矿化度 $35.74 \mathrm{~g} / \mathrm{L}^{[15]}$, 溶解氧 $105.64 \mathrm{mg} / \mathrm{L}, \mathrm{pH}$ 值 $8.38^{[14]}$. 据德令哈市气象站 $(1960-2016$ 年) 气候资料显示 (图 $1 \mathrm{~b})$ : 区域多年平均气温为 $4^{\circ} \mathrm{C}, 7$ 月份最热, 平均气温为 $16.7^{\circ} \mathrm{C}, 1$ 月份最冷, 平均气温为 $-11^{\circ} \mathrm{C}$, 降水季节性 明显, 主要集中在 5-9 月, 多年夏季平均降水量为 180 200 mm, 冬季降水量约为 17 22 mm, 多年平均蒸发 量约 $1950 \mathrm{~mm}$; 托素湖自动气象站(1)记录了湖区 2017 年和 2018 年 1-10 月的气温和降水(图 1c,2 年有记录 月份的平均值), 虽缺少 11 月和 12 月数据 (缺测), 但可以看出湖区的温度和降水与德令哈站类似. 湖区的 植被类型为荒漠草原, 主要有藜科 (Chenopodiaceae)、麻黄 (Ephedra)、白刺 (Nitraria) 和菊科 (Compositae) 等 荒漠植被 ${ }^{[8]}$.

\section{2 材料与方法}

课题组于 2015 年 8 月使用重力钻在托素湖获得短钻岩芯 3 根 (位置如图 1a), 分别为 TSL15S1 (水深 1 $\mathrm{m}$ ), TSL15S3(水深 $16.5 \mathrm{~m}$ ) 和 TSL15S7 (水深 $16.5 \mathrm{~m}$ ), 长度分别为 $33 、 38$ 和 $40 \mathrm{~cm}$; 于 2017 年 8 月在水深 $25 \mathrm{~m}$ 处获得短钻岩芯 1 根 (TSLG17, 长 $55 \mathrm{~cm}$ ). 4 根短钻岩芯均以 $5 \mathrm{~mm}$ 间隔分样, 用自封袋密封后编号称 重、冷冻干燥备用.

利用 ${ }^{210} \mathrm{~Pb}$ 和 ${ }^{137} \mathrm{Cs}$ 方法分别测定 4 个岩芯沉积物的年代. 将 $2 \sim 3 \mathrm{~g}$ 冷冻干燥后的样品装人特制透明塑 料样品管 (直径 $\times$ 高 $=14.5 \mathrm{~mm} \times 40 \mathrm{~mm}$ ) 内盖紧, 静置 20 天以上, 使得 ${ }^{226} \mathrm{Ra}$ 与 ${ }^{222} \mathrm{Rn}$ 达到永久放射性准平衡状 态. 然后用堪培拉欧洲系统测量公司生产的高纯锗 $\gamma$ 能谱仪 (井型探测器) 进行 ${ }^{210} \mathrm{~Pb}$ 和 ${ }^{137} \mathrm{Cs}$ 核素比活度测 试,每个样品测试时间均为 $86400 \mathrm{~s}$, 使用 Genie-2000 Gamma Option 谱分析软件分析测得的数据.

使用气量法 ${ }^{[16]}$ 对碳酸盐含量进行测试, 并利用下式计算碳酸盐含量:

$$
\mathrm{CaCO}_{3}(\%)=\frac{0.16 \times 0.75 P \cdot V}{m(273+T)} \times 100
$$

式中, $P$ 为当时的大气压强, $\mathrm{hPa} ; V$ 为生成的 $\mathrm{CO}_{2}$ 气体体积, $\mathrm{mL} ; m$ 为样品质量, $\mathrm{g} ; T$ 为温度, ${ }^{\circ} \mathrm{C}$.

使用 Mastersizer 2000 激光粒度仪对沉积物样品粒度指标进行测试, 测试前需先对样品进行去除有机质 和碳酸盐的处理, 前处理方法采用湖泊沉积物标准处理方法 ${ }^{[17]}$, 粒径测量范围在 $0.02 \sim 2000.00 \mu \mathrm{m}$, 测量误

(1) 资料来源: 青海省气象局. 

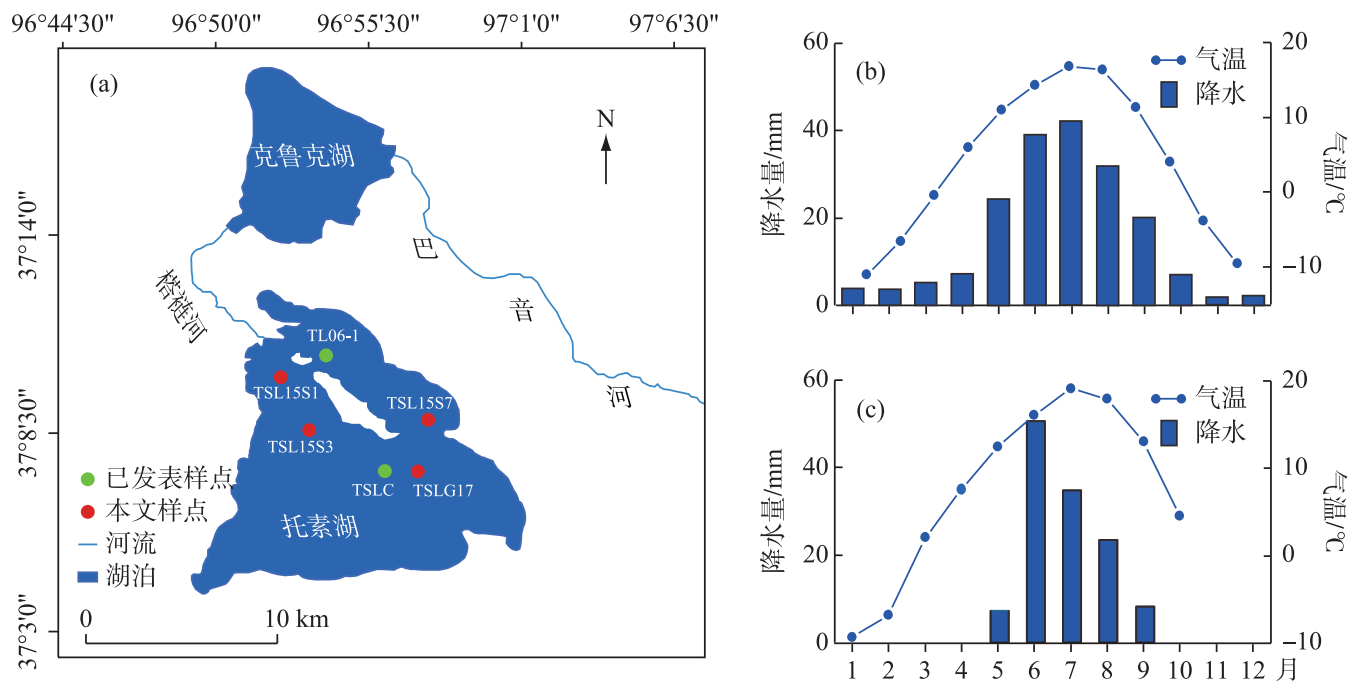

图 1 研究区及采样点位置 (a)、德令哈气象站(1960-2016 年) (b)、

托素湖自动气象站(2017-2018 年) (c) 月均气温、降水数据

Fig. 1 Study area and the cores site (a), monthly temperature and precipitation from Delingha Station (b) and

Toson Station (c) (data from Delingha meteorological station during 1960-2016 AD,

and Lake Toson automatic meteorological station during 2017-2018 AD)

差小于 $0.2 \%$.

利用烧失法对沉积物中有机质含量进行测试. 具体步骤如下: (1) 将 $10 \mathrm{~mL}$ 坩埚在 $600^{\circ} \mathrm{C}$ 的马弗炉中, 煅 烧半小时, 去除有机质. 放人干燥血.中待冷却后用万分位天平秤取其重量, 记 $M_{1}$; (2) 称取大约 $0.5 \mathrm{~g}$ 干样放 人坩埚内, 在 $105^{\circ} \mathrm{C}$ 下烘干 $12 \mathrm{~h}$ 冷却后称重, 记 $M_{2}$; (3) 将坩埚置于马弗炉中, 升温至 $550^{\circ} \mathrm{C}$ 烧 $4 \mathrm{~h}$, 冷却后称 重记 $M_{3}$. 用 $550^{\circ} \mathrm{C}$ 的烧失量值代表有机质含量,记作 $\mathrm{OM}(\%)$, 计算公式为:

$$
\mathrm{OM}(\%)=\frac{\left(M_{2}-M_{3}\right)}{\left(M_{2}-M_{1}\right)} \times 100
$$

以上实验均在兰州大学西部环境教育部重点实验室完成.

\section{3 结果}

\section{1 年代序列的建立}

根据放射性活度测试结果, 4 根岩芯的 ${ }^{210} \mathrm{~Pb}$ 总活度和 ${ }^{226} \mathrm{Ra}$ 活度平衡深度分别在 $16 、 20 、 18$ 和 $11 \mathrm{~cm}$, 此 深度以下, 过剩 ${ }^{210} \mathrm{~Pb}\left({ }^{210} \mathrm{~Pb}_{\mathrm{ex}}\right)$ 活度为零, 不再具有计年意义, 其 ${ }^{137} \mathrm{Cs}$ 及 ${ }^{210} \mathrm{~Pb}_{\mathrm{ex}}$ 结果如图 2 所示. 各孔沉积岩 芯 ${ }^{137} \mathrm{Cs}$ 活度均有较为明显的单一峰值, ${ }^{210} \mathrm{~Pb}_{\mathrm{ex}}$ 活度均存在不同程度的波动.

目前, 基于 ${ }^{210} \mathrm{~Pb}_{\text {ex }}$ 活度计算沉积物年代的方法主要包括恒定沉积通量模式 CIC 和恒定放射性通量模式 $\mathrm{CRS}^{[18]}$, 其中 CRS 模式是基于 ${ }^{210} \mathrm{~Pb}$ 沉积通量不变,沉积速率可能发生改变的情况. 我们先采用 CRS 模式建 立了托素湖 4 孔沉积岩芯年代序列 (图 3), 结果发现, 4 孔沉积岩芯 ${ }^{210} \mathrm{~Pb}$ 年代均偏离 ${ }^{137} \operatorname{Cs} 1963$ 年时标, 定年 结果存在误差. 周爱锋等在研究柴达木盆地西北部苏干湖的 ${ }^{210} \mathrm{~Pb}$ 和 ${ }^{137} \mathrm{Cs}$ 年代时也发现, ${ }^{137} \mathrm{Cs}$ 时标 (公元 1963 年) 与 ${ }^{210} \mathrm{~Pb}$ 年代 (公元 1972 年)之间存在差异, 且不能用 $\mathrm{Cs}$ 元素的迁移来解释 ${ }^{[19]}$. 同时, 有研究表明, 虽然沉积物中 ${ }^{137} \mathrm{Cs}$ 存在一定扩散迁移, 但其峰值位置不会发生改变 ${ }^{[20]}$. 故我们采用另一种常用的方法一 复合模式 ${ }^{[21-22]}$, 根据 ${ }^{137} \mathrm{Cs}$ 的 1963 年时标深度将沉积岩芯划分为上下两部分, 分别进行沉积岩芯年代计算, 结果见图 3. 将 CRS 模式和复合模式计算的年代结果进行对比发现,其中 2 种模式得出的 TSL15S1、TSL15S3 与 TSLG17 孔年代结果大体一致, 而复合模式计算的 TSL15S7 孔年代结果明显较 CRS 模式结果偏老, 因此, 

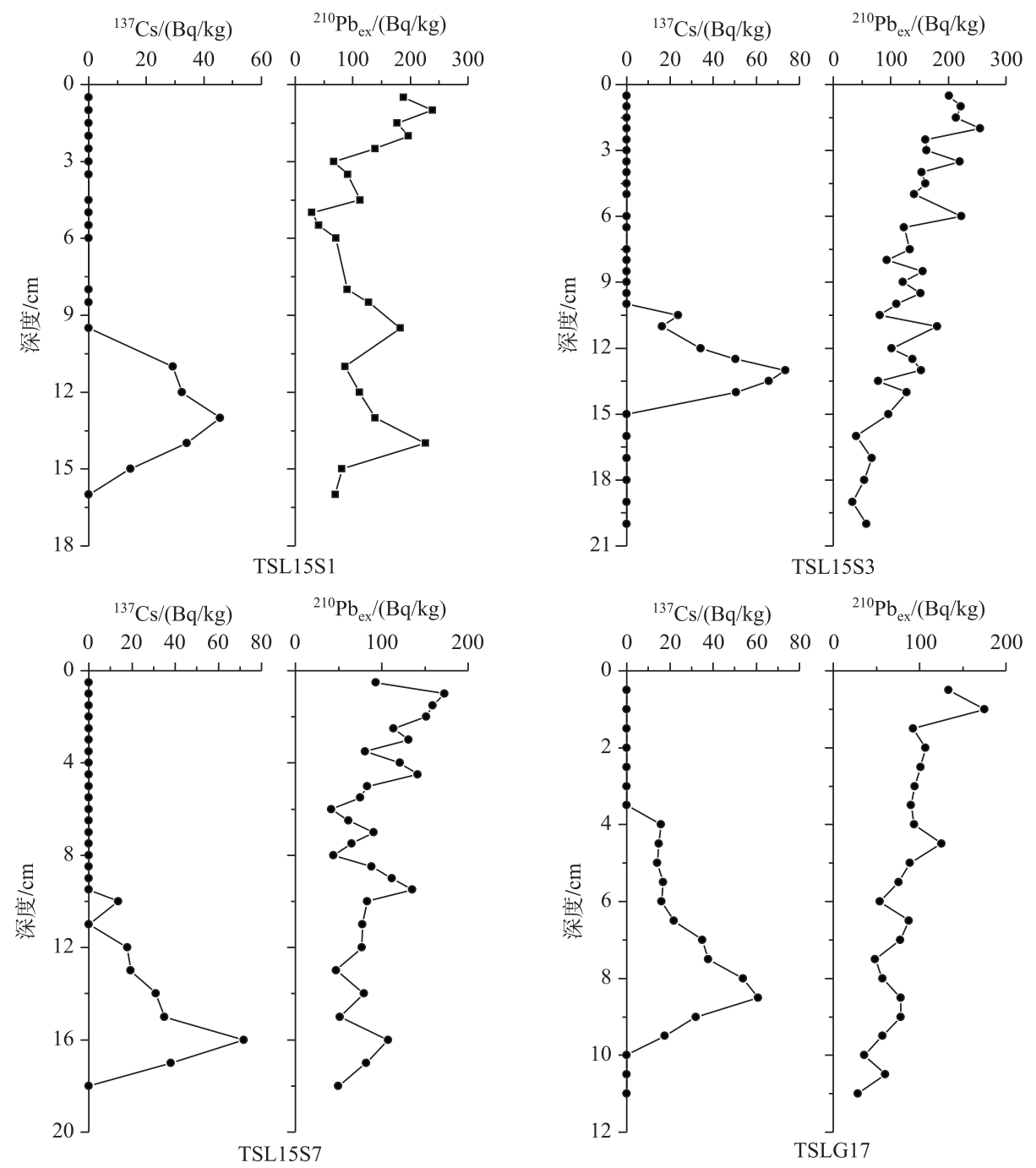

图 2 TSL15S1、TSL15S3、TSL15S7 与 TSLG17 孔岩芯沉积物 ${ }^{137} \mathrm{Cs}$ 及 ${ }^{210} \mathrm{~Pb}_{\mathrm{ex}}$ 的垂直分布

Fig. 2 Vertical distributions of excess ${ }^{137} \mathrm{Cs}$ and ${ }^{210} \mathrm{~Pb}_{\mathrm{ex}}$ of the TSL15S1, TSL15S3, TSL15S7 and TSLG17

本文最终采用复合模式的计算结果作为 TSL15S1、TSL15S3、TSL15S7 和 TSLG17 孔的年代数据, 其底部年代 分别为 $1911 \mathrm{AD} 、 1895 \mathrm{AD} 、 1934 \mathrm{AD}$ 和 $1901 \mathrm{AD}$.

\section{2 托素湖不同位置沉积物近 100 年来代用指标变化特征}

托素湖不同位置沉积岩芯沉积物平均粒径变化如图 4 所示 (其中,TSLC 孔 ${ }^{[14]}$ 的年代采用经过复合模式 校正后的年代,下同). 空间上距离河口较近的浅水区的 TSL15S1 孔的平均粒径变化最大(范围: 26.83 $111.17 \mu \mathrm{m}$, 平均值: $57.14 \mu \mathrm{m}$ ), 湖岛西侧中等水深 $16.5 \mathrm{~m}$ 处的 TSL15S3 孔平均粒径变化次之(范围: 29.07 71.45 $\mu \mathrm{m}$; 平均值 $46.17 \mu \mathrm{m}$ ), 而湖岛东侧与 TSL15S3 相同水深处的 TSL15S7 孔的平均粒径的变化范 围 (18.01 50.90 $\mu \mathrm{m}$ ) 和平均值 $(27.39 \mu \mathrm{m}$ ) 都较西侧小得多; 湖泊偏南部的深水区 TSLG17 以及 TSLC 孔平 均粒径变化较小 (范围分别为 19.39 32.65 和 24.24 47.48 $\mu \mathrm{m}$; 平均值分别为 27.72 和 $32.89 \mu \mathrm{m}$ ). 可以看 出, 托素湖这几个岩芯的平均粒径整体上随离岸距离和水深的增加而逐渐减小, 符合湖泊沉积物粒度变化 的一般特征. 同时, 位置临近的岩芯( TSL15S1 和 TSL15S3; TSL15S7、TSLG17 和 TSLC) 沉积物平均粒径变化 

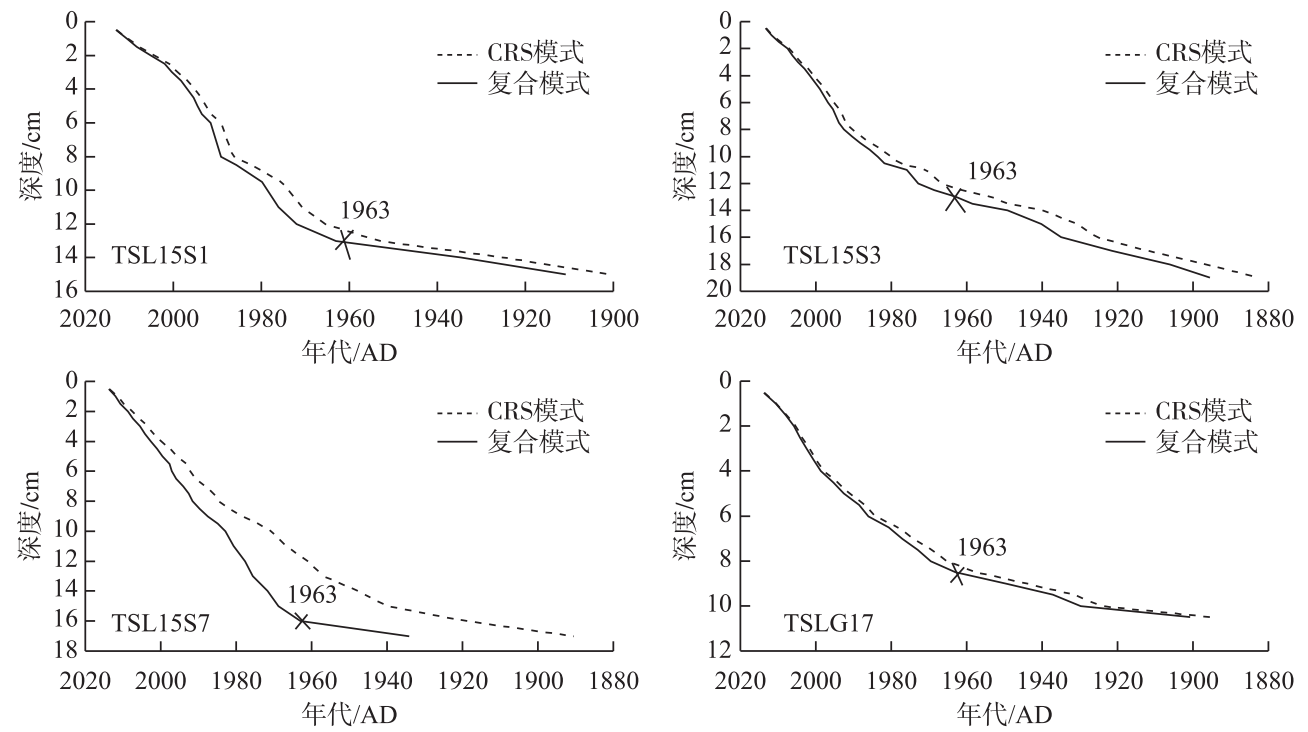

图 3 托素湖 4 个短钻岩芯年代一深度模型 (图中 1963 为 ${ }^{137} \mathrm{Cs}$ 时标)

Fig.3 The age-depth model of the four short cores from Lake Toson (the 1963 represented a ${ }^{137}$ Cs marker)

较为一致,相同水深处 TSL15S3 和 TSL15S7 孔平均粒径的差异反映了湖岛东西侧沉积环境差异, 西侧水动 力大于东侧. 因此, 从接近河口的浅水区至远离河口的深水区, 沉积物粒度的这种差别反映了湖泊内水动力 逐渐减弱的基本特征, 深水区沉积物粒径变化幅度小, 没有了类似浅水区沉积物粒径的大幅波动.

平均粒径 $/ \mu \mathrm{m}$

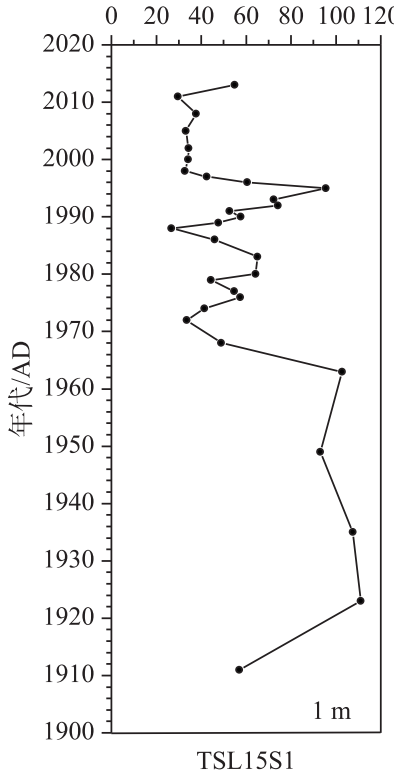

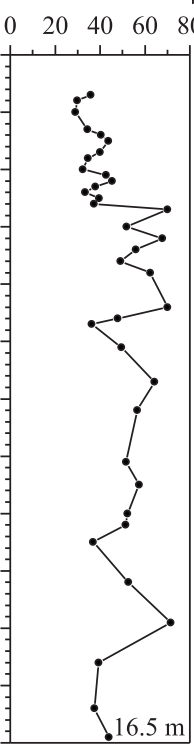

TSL15S3

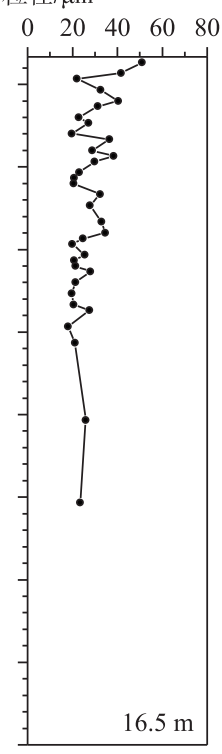

TSL15S7

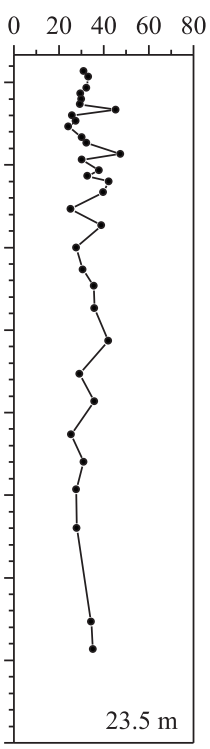

TSLC

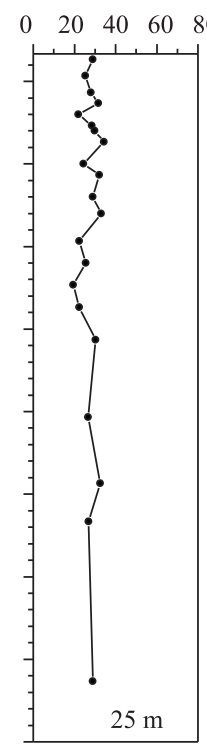

TSLG17

图 4 托素湖不同位置岩芯沉积物平均粒径 (图中 $1 \mathrm{~m}$ 为采样点水深,下同)

Fig.4 Mean grain-size of the cores from five sites in Lake Toson (the $1 \mathrm{~m}$ represents the water depth of cores, the same below) 
按黏土 $(<4 \mu \mathrm{m})$ 、粉砂 $(4 \sim 63 \mu \mathrm{m})$ 和砂 $(>63 \mu \mathrm{m}) 3$ 个组分划分的托素湖各岩芯粒度组分如图 5. 可以 看出,不同位置的 4 孔沉积物的黏土组分含量变化不大 (平均值分别为 $15.11 \% 、 18.69 \% 、 19.66 \%$ 和 $20.35 \%$ ), 且具有相似性, 即 $1970 \mathrm{~s}$ 中期至 1990s 中期黏粒含量减少, 1990s 中期以后呈增加趋势. 不同位置 的沉积物均以粉砂组分为主, 平均值分别为 58.65\%、59.67\%、71.19\% 和 $69.62 \%$; 位置临近的岩芯( TSL15S1 与 TSL15S3; TSL15S7 与 TSLG17) 沉积物的粉砂组分含量变化较为一致; 砂粒组分含量在浅水区 ( TSL15S1 孔平均值为 $26.24 \%$ ) 最高, 同为 $16.5 \mathrm{~m}$ 水深湖岛西侧的 TSL15S3 较湖岛东侧的 TSL15S7 孔砂粒组分含量高 (平均值分别为 $21.63 \%$ 和 $9.15 \%$ ), 这与平均粒径的特征一致; 深水区 TSLG17 砂粒组分含量平均值接近 $10 \%$, 砂粒组分含量在各孔的变化趋势不同,相似性低.

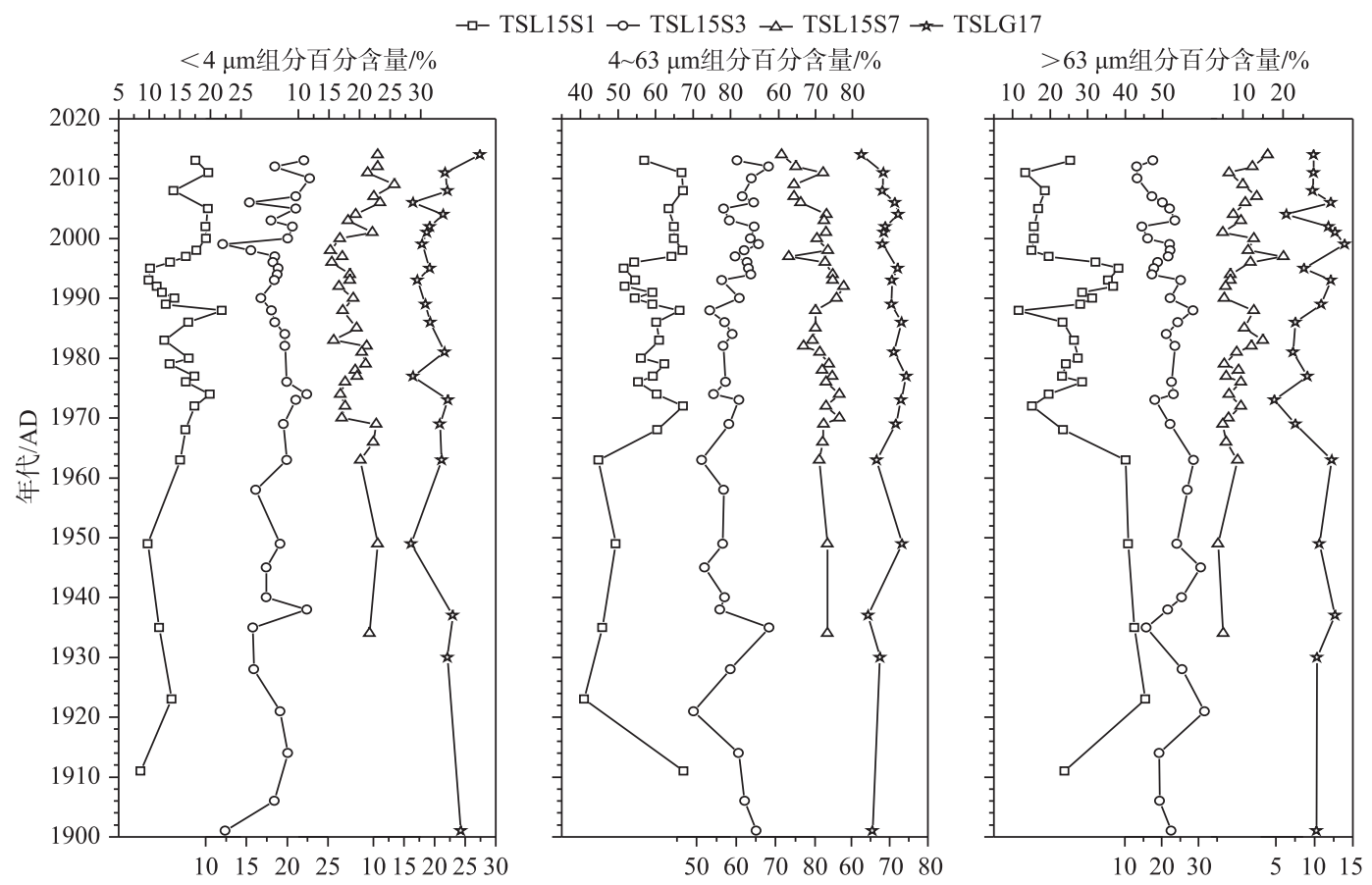

图 5 托素湖不同位置沉积岩芯沉积物粒度组分

Fig.5 Grain size composition of sediments of the cores from four sites in Lake Toson

托素湖不同位置沉积岩芯沉积物碳酸盐和有机质含量变化如图 6 所示. 由于部分岩芯剩余样品量不足 以分析有机质含量, 故仅 3 个岩芯有有机质含量(图 6). TSL15S1 孔( 水深 $1 \mathrm{~m}$ ) 有机质含量( 范围: 14.08\% $40.07 \%$; 平均值: $25.36 \%$ ) 和碳酸盐含量 (范围: $22.42 \% \sim 70.59 \%$; 平均值: $41.29 \%$ ) 均较高, 且变化波动较大; TSL15S3 孔和 TSL15S7 孔 ( 水深 $16.5 \mathrm{~m}$ ) 碳酸盐含量变化范围接近 ( 分别为 $17.40 \% \sim 51.97 \%$ 和 $16.70 \% \sim$ $46.37 \%$ ), 平均值分别为 $33.15 \%$ 和 $29.06 \%$; TSL15S7 孔的有机质含量 (范围: $10.84 \% \sim 25.6 \%$; 平均值: $17.07 \%$ ) 较浅水区有所降低; TSLG17 孔 (水深 $25 \mathrm{~m}$ ) 和 TSLC 孔 (水深 $23.5 \mathrm{~m}$ ) 岩芯的沉积物碳酸盐含量最 低, 且变化幅度最小 $(21.38 \% \sim 38.94 \%$ 和 $19.64 \% \sim 35.82 \%)$, 平均值分别为 $28.8 \%$ 和 $27.46 \%$. 深水区 TSLG17 孔有机质含量进一步降低, 变化范围在 $12.12 \% \sim 22.26 \%$ 之间, 平均值为 $15.93 \%$. 因此, 托素湖碳酸盐和有机 质含量分布的一个显著特点是水深越浅, 沉积物碳酸盐和有机质含量越高, 且相同水深处沉积物碳酸盐含 量基本相同; 另一个特点是浅水区碳酸盐或有机质含量波动幅度大于深水区 (图 6 中横坐标刻度比例相 同), 例如碳酸盐含量自 $1970 \mathrm{AD}$ 以来升高并于 $2000 \mathrm{AD}$ 后降低, 虽然各孔均有体现, 但深水区变化幅度小 得多.

从有机质含量和碳酸盐含量的关系 (图 6) 看, 3 个岩芯沉积物中碳酸盐含量和有机质含量随时间变化 趋势类似, 即同时升高或降低. 其中 TSL15S1 和 TSL15S7 孔有机质和碳酸盐的相关性达到显著正相关, 深水 


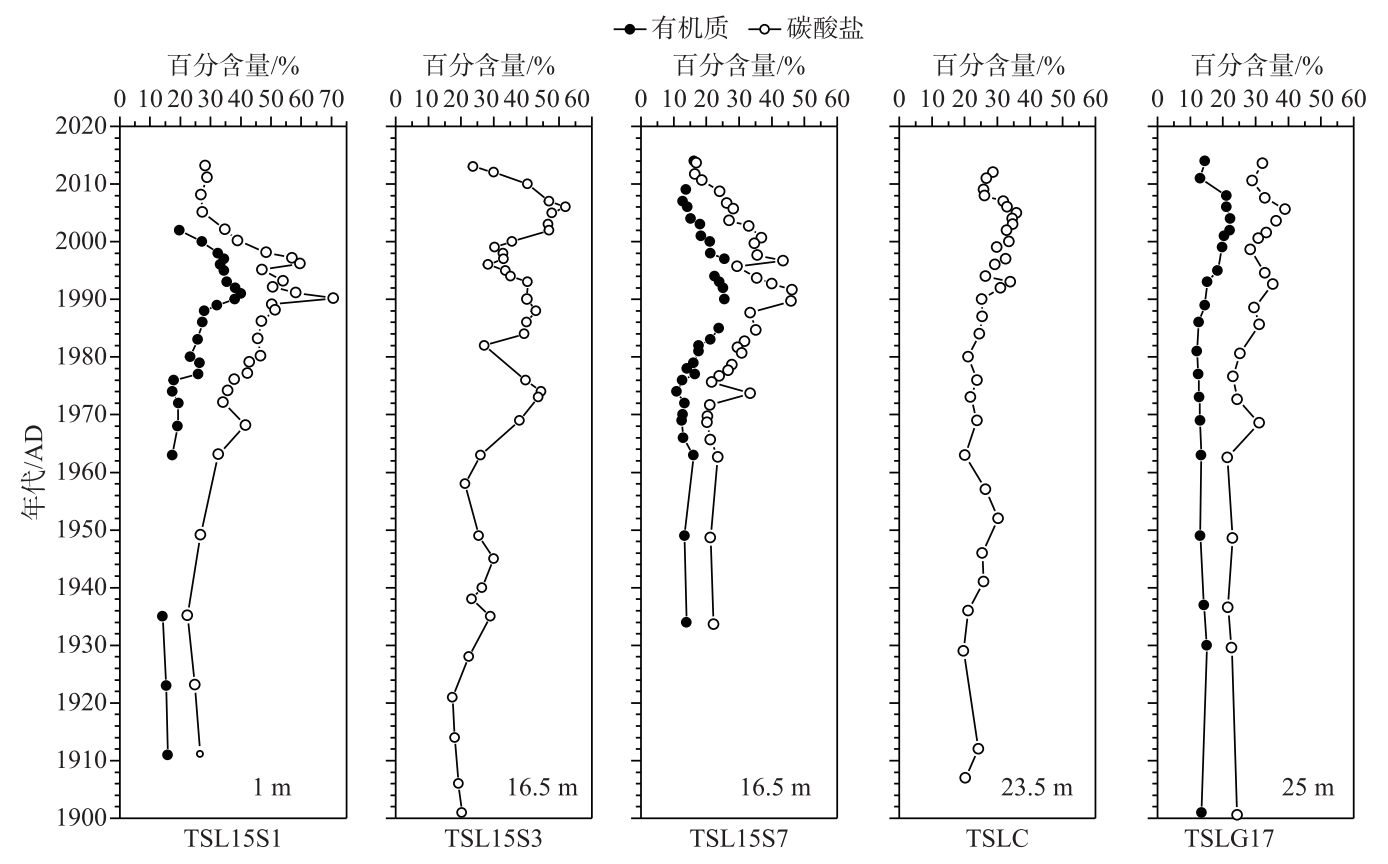

图 6 托素湖不同沉积岩芯碳酸盐和有机质含量

Fig. 6 Contents of carbonate and organic matter of the cores from five sites in Lake Toson

区 TSLG17 孔的有机质和碳酸盐含量相关性较弱.

\section{4 讨论}

\section{1 不同位置沉积岩芯指标变化及其环境意义}

上述湖泊内不同位置沉积物粒度的差异性 (图 4 5) 证实, 同一湖泊不同位置沉积岩芯的沉积物粒度大 小会有明显的不同 ${ }^{[23-24]}$. 在湖泊面积、水深都较大的情况下, 深水区 (如大于 $20 \mathrm{~m}^{[25]}$ ) 沉积物的粒度变化范 围很小,与相距较远位置的沉积物粒度已不具备可比性. 例如, 托素湖深水区沉积物粒度在短时间尺度上 (过去 100 年, 图 4)、小幅度的波动仅体现了沉积环境 (水体动力状况) 的细微波动. 湖泊深水区沉积物平均 粒径稳定, 实际研究中经常使用沉积物粒度组分 ${ }^{[23,25]}$ 或计算粒度特征参数. 例如, 青海湖, 深水区沉积物平 均粒径较小 (16 $\mu \mathrm{m}$ 左右), 过去一万年以来也变化不大, 实际研究中使用粒度资料计算粒度参数并与已知 的气候变化序列进行对比 ${ }^{[26]}$; 纳木错水深 $60 \mathrm{~m}$ 的岩芯沉积物平均粒径的变化不及其粒度组分 $(<4 \mu \mathrm{m} 、 4 \sim$ $63 \mu \mathrm{m}$ 和 $>63 \mu \mathrm{m}$ ) 的指示意义明确 ${ }^{[27]}$. 托素湖沉积岩芯粒度各组分中 (图 5), 仅黏土组分变化具有相似 性, 可能代表了可长时间悬浮的悬移组分; 粉砂和砂组分在浅水区 (TSL15S1) 和深水区 (TSLG17) 均不具备 可比性. 托素湖的情况表明, 湖泊沉积物的平均粒径或主要组分 (如粉砂) 含量的大幅度波动出现在浅水区, 深水区这两个指标都很稳定. 因此, 使用湖泊内单个点沉积物粒度指标时, 其环境意义有限, 需要结合其他 多指标相互印证才可确认.

尽管干旱区湖泊沉积物中有风成组分 (风力搬运), 但在没有详细的观测进行量化的情况下,用特定组 分的含量 ${ }^{[28-30]}$ 或者各类端元数学模型分离出来的组分 ${ }^{[26,31-32]}$ 来代表风力输人的成分的研究, 都需要谨慎. 因此,本文对沉积物中风力搬运贡献的组分未作讨论.

碳酸盐含量在时间上变化的相似性(图 6) 则表明,湖泊水体混合相对均匀,水体化学特征在湖泊不同位 置大致相同, 并通过水体析出的碳酸盐沉淀保存下来. 除干旱地区湖泊外, 在其他地区也较为常见,如星云 湖 7 个位置的钻孔碳酸盐含量 (或 XRF 扫描的 $\mathrm{Ca}^{2+}$ 强度) 均具有较好的对应关系 ${ }^{[33]}$, 因此, 湖泊中不同位置 的碳酸盐含量可以用来作为岩芯对比 (接) 的依据, 并用来反映区域气候环境变化. 目前碳酸盐含量主要用 
来反映区域有效湿度 ${ }^{[3,34]}$ 、湖泊生产力 ${ }^{[35-36]}$ 等. 在青藏高原湖泊沉积物中, 碳酸盐含量升高常意味着湖泊水 位降低, 反映流域内干旱程度增加 ${ }^{[34,37-38]}$. 当湖泊沉积物碳酸盐含量较低时, 需要证明其来源为湖泊自 生 ${ }^{[39]}$. 在包括青藏高原在内的干旱和半干旱区, 封闭湖泊沉积物碳酸盐含量较高, 且以湖泊自生碳酸盐为 主, 这可以从几个方面得到验证: 首先, 中东亚地区(没有石灰岩分布地区) 表土碳酸盐含量在黄土地区表土 最高 ( $10 \%$ 左右), 其他地区均在 $5 \%$ 以下 ${ }^{[40]}$; 其次, 干旱区湖泊 (新疆巴里坤湖) 湖边收集到的风尘粉尘的碳 酸盐含量平均接近 $10 \%$, 且在湖泊中由风力搬运的外源碳酸盐不足碳酸盐总量的 $10 \%^{[41]}$; 第三, 高原上湖 泊深钻孔揭示, 河流沉积物碳酸盐含量很低 (不足 5\%), 但形成稳定的封闭湖泊后, 沉积物中的碳酸盐含量 显著升高 ${ }^{[37]}$, 表明是由湖泊水体沉淀的自生碳酸盐. 上述几种情况表明, 以碎屑碳酸盐形式被流水或风力 搬运人湖的碳酸盐总含量不超过 $10 \%$, 当湖泊沉积物中碳酸盐含量大于 $20 \%$ 时, 即意味着沉积物中的碳酸 盐以湖泊自生碳酸盐为主, 可以用于古气候环境变化研究. 托素湖碳酸盐含量多大于 $20 \%$, 因此可以认为以 湖泊自生碳酸盐为主,其含量的变化指示了区域有效湿度的变化.

湖泊沉积物中有机质含量虽然受到到内源、外源等因素的影响, 但作为流域和湖泊生产力变化的指标, 仍被广泛应用于古气候研究中 ${ }^{[42-43]}$. 湖泊不同位置有机质含量变化趋势 (图 6) 的相似性表明, 有机质含量 能有效地指示流域和湖泊的生产力. 陆源有机质主要由湖区降水形成的地表径流带人, 湖区降水增加, 流域 内植被生产力提高, 生物量增加, 湖泊有机质含量增加; 反之, 流域降水量减少, 植被生产力降低, 生物量减 少, 湖泊有机质含量减少 ${ }^{[44]}$. 对于托素湖这样处于干旱区的湖泊来说, 陆源有机质含量较少, 沉积物中的有 机质大多为内源有机质, 其含量指示了湖泊生产力的变化. 托素湖沉积物有机质含量整体较高, 指示湖泊生 产力较高.

碳酸盐与有机质含量的正相关性表明, 湖泊浅水区碳酸盐可能更多地受到湖泊水生 (浮游或沉水) 植物 生产力的影响: 浅水区水体光照、温度条件好, 浮游和沉水藻类光合作用促进水体碳酸盐沉淀 ${ }^{[39]}$, 所以浅水 区的碳酸盐含量也较高. 深水区下部水温低、光线减弱, 藻类光合作用减弱, 有机质含量降低, 碳酸盐与有机 质含量的相关关系减弱.

\section{2 区域过去几十年来湖泊沉积记录的解释}

托素湖各孔沉积物粒度 (平均粒径或组分含量) 变化虽符合水动力在湖泊内随水深增加而变弱的一按 规律, 但粒度指标与其他指标的变化都不太一致,所以该湖粒度变化代表了局地的沉积环境状况,深水区沉 积环境稳定. 因此, 我们使用深水区地球化学指标, 与有器测资料时段以来的气象观测资料对比, 分析过去 几十年的湖泊沉积记录的环境变化.

将深水区有机质含量 (图 7a) 和碳酸盐含量 (图 7b) 的结果与托素湖已发表的 TSLC 孔碳酸盐氧同位 素 ${ }^{[14]}$ 和 TL06-1 孔孢粉的 A/C 比值 ${ }^{[8]}$ 、树轮重建的区域降水 ${ }^{[45]}$ 以及德令哈气象站数据进行对比分析 (图 7) 发现, 湖泊沉积物中这几个反映区域有效湿度的代用指标(图 $7 \mathrm{~b} \sim \mathrm{d}$ ) 变化基本一致( 碳酸盐含量增加、碳酸 盐氧同位素偏正以及孢粉的 $\mathrm{A} / \mathrm{C}$ 比值减小), 即 $1960 \mathrm{AD}$ 以来有效湿度减小, 这与同期器测资料中的相对湿 度 (图 7e) 变化趋势也吻合, 但是与树轮重建的区域降水量 (图 7f) 及同期器测的降水量 (图 7g) 呈增加的趋 势不一致.

上述这种沉积记录的有效湿度变化与实际降水量之间存在的差异, 值得引起注意. 通常我们认为可能 是由于全球变暖, 区域温度升高, 导致蒸发量增加所致. 但同期的器测潜在蒸发量 (图 $7 \mathrm{~h}$ ) 却在减小. 为了进 一步确认, 我们使用彭曼公式计算了同时期湖面的蒸发量, 结果显示也是减小的 (图 7i). 尽管从月资料中蒸 发量与温度的关系看, 温度高的时候蒸发量在增加 ${ }^{[6]}$, 但年资料 (图 $7 \mathrm{j} 、 \mathrm{~h}$ ) 显示温度在增加, 蒸发量在减小. 付霞等在研究 TSLC 孔时认为德令哈附近人类活动在流域内用水量增加导致了实际人湖水量减少 ${ }^{[14]}$, 即虽 然区域的实际降水量在增加, 但是湖泊沉积记录的区域有效湿度却在减小. 因此, 湖泊沉积记录与器测记录 不一致时,考虑可能是由于人类活动影响了流域的水文过程.

\section{5 结论}

本文通过分析托素湖不同位置的沉积岩芯的沉积物粒度、有机质及碳酸盐含量, 并与托素湖指示意义 明确的自生碳酸盐氧同位素 $\left(\delta^{18} \mathrm{O}\right)$ 和狍粉 $\mathrm{A} / \mathrm{C}$ 值以及树轮重建的区域降水量、器测资料进行对比, 得出以 


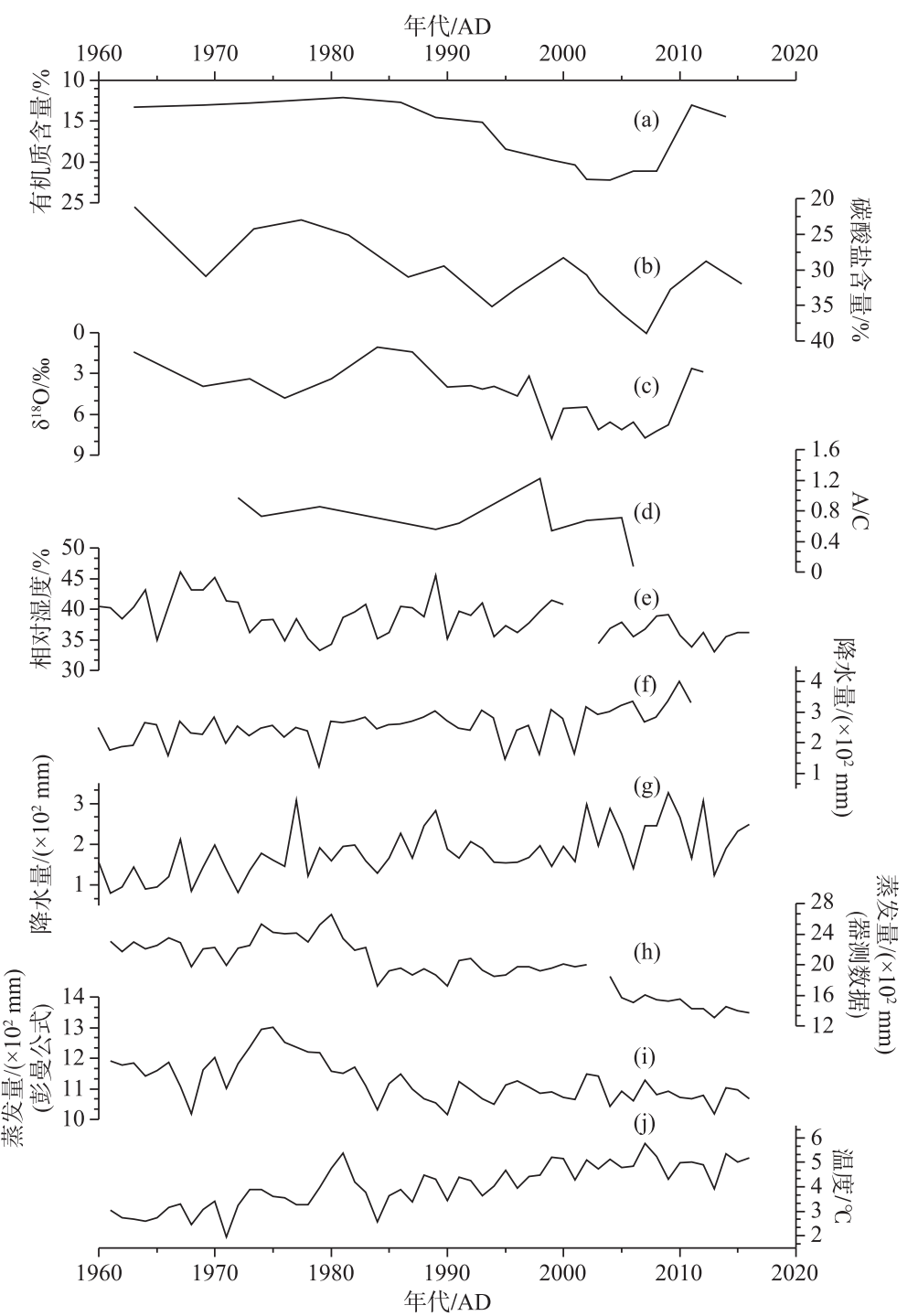

图 7 代用指标与区域记录、器测资料对比:

(a) TSLG17 孔有机质含量; (b) TSLG17 孔碳酸盐含量; (c) TSLC 孔自生碳酸盐氧同位素 ${ }^{[14]}$;

(d) TL06-1 孔狍粉 A/C 值 ${ }^{[8]}$; (e) 年均相对湿度; (f) 树轮重建的降水 ${ }^{[45]} ;(\mathrm{g})$ 年平均降水; ( h ) 年蒸发量;

(i) 彭曼公式计算的蒸发量; ( j ) 年均气温(所有器测数据均来自德令哈市气象站 $(1960-2016$ 年))

Fig.7 Comparison of proxies with other core records of Lake Toson and instrumental data:

(a) organic matter content of TSLG17, (b) carbonate content of TSLG17, (c) carbonate $\delta^{18} \mathrm{O}$ from TSLC ${ }^{[14]}$, (d) A/C ratio of TL06-1 ${ }^{[8]}$, (e) relatively humidity,

(f) the reconstructed regional precipitation based on tree ring $^{[45]},(\mathrm{g})$ annual average precipitation, (h) annual evaporation, (i) evaporation calculated by Penman formula, (j) annual average temperature (all instrumental data were taken from Delingha meteorological station during 1960-2016 AD)

下结论:

1 ) 湖泊内相近的沉积岩芯沉积物粒度变化具有一致性,但相距较远的岩芯之间粒度变化差异较大. 浅 水区的沉积物粒度 (粒径) 在短时间尺度上可以变化很大; 而深水区的沉积环境比较稳定,在百年尺度上的 
变化都不明显. 因此,托素湖粒度指标仅指示取样点沉积环境, 与气候变化关系不明显.

2) 湖泊沉积物中有机质和碳酸盐总体变化趋势一致,有较好的相关性, 且浅水区相关性更好, 表明碳酸 盐可能受到湖泊生产力的影响. 湖泊内碳酸盐和有机质含量变化趋势的一致性表明在这类湖泊中选择地球 化学指标恢复区域气候环境更可靠.

3 ) 湖泊不同位置沉积岩芯碳酸盐含量与碳酸盐 $\delta^{18} \mathrm{O}$ 以及孢粉 $\mathrm{A} / \mathrm{C}$ 值的变化均具有一致性, 反映流域 有效湿度的变化, 但 $1960 \mathrm{AD}$ 以后与器测资料显示的降水量增加、蒸发量减小并不一致, 可能是受到人类活 动的影响.

致谢: 兰州大学付霞、王林、崔雪莉以及华中师范大学梅换参加了湖泊岩芯钻探工作, 在此一并致谢!

\section{6 参考文献}

[ 1 ] Wang SM, Zhang ZK. New advance of lake sediments and environmental changes study in china. Chinese Science Bulletin, 1999, 44(6) : 579-587. [王苏民, 张振克. 中国湖泊沉积与环境演变研究的新进展. 科学通报, 1999, 44(6): 579-587.]

[ 2 ] Liu J, Wang Y, Li T et al. Comparison of grain-size distributions between nearshore sections and a deep-water sediment core from Dali Lake, North China, and inferred Holocene lake-level changes. Journal of Paleolimnology, 2016, 56(2/3) : 123-135.

[ 3 ] Cao JT, Xu AX, Wang SM et al. Carbonate content record of climate and environment changes from lacustrine core in Daihai Lake, Inner Mongolia. Transactions of Oceanology and Limnology, 1999, 4(4): 21-26. [曹建廷, 徐爱霞, 王苏 民等. 内蒙岱海湖岩芯碳酸盐含量变化与气候环境演化. 海洋湖沼通报, 1999, 4(4): 21-26.]

[ 4 ] Morinaga H, Itota C, Isezaki N et al. Oxygen-18 and carbon-13 records for the last 14,000 years from Lacustrine carbonates of Siling-Co (Lake) in the Qinghai-Tibetan Plateau. Geophysical Research Letters, 1993, 20(24) : 2909-2912.

[ 5 ] Li X, Liu W, Xu L. Stable oxygen isotope of ostracods in recent sediments of Lake Gahai in the Qaidam Basin, northwest China: The implications for paleoclimatic reconstruction. Global and Planetary Change, 2012, 94/95: 13-19.

[ 6 ] Li X, Zhou X, Liu W et al. Carbon and oxygen isotopic records from Lake Tuosu over the last 120 years in the Qaidam Basin, Northwestern China: The implications for paleoenvironmental reconstruction. Global and Planetary Change, 2016, 141 : $54-62$.

[ 7 ] Zhao Y, Yu Z, Chen F et al. Sensitive response of desert vegetation to moisture change based on a near-annual resolution pollen record from Gahai Lake in the Qaidam Basin, northwest China. Global and Planetary Change, 2008, 62 (1/2) : 107-114.

[ 8 ] Zhao Y, Yu Z, Liu X et al. Late Holocene vegetation and climate oscillations in the Qaidam Basin of the northeastern Tibetan Plateau. Quaternary Research, 2010, 73(1) : 59-69.

[ 9 ] Li Y, Jin ZD. Seasonal and interannual variations in abundance and oxygen-carbon isotopic compositions of ostracod shells from Lake Qinghai and their controlling factors: A case study on the sediment trap. Journal of Earth Environment, 2013, 4 (3) : 1328-1337. [李燕, 金章东. 青海湖介形虫壳体丰度与氧碳同位素的季节和年际变化及其控制因素一一来自 沉积物捕获器的研究. 地球环境学报, 2013, 4(3): 1328-1337.]

[10] Wang J, Zhu L, Nishimura M et al. Spatial variability and correlation of environmental proxies during the past 18,000years among multiple cores from Lake Pumoyum Co, Tibet, China. Journal of Paleolimnology, 2009, 42(3) : 303-315.

[11] Opitz S, Wünnemann B, Aichner B et al. Late Glacial and Holocene development of Lake Donggi Cona, north-eastern TibetanPlateau, inferred from sedimentological analysis. Palaeogeography, Palaeoclimatology, Palaeoecology, 2012, 337/ 338: $159-176$.

[12] Wünnemann B, Wagner J, Zhang Y et al. Implications of diverse sedimentation patterns in Hala Lake, Qinghai Province, China for reconstructing Late Quaternary climate. Journal of Paleolimnology, 2012, 48(4) : 725-749.

[13] Ling Y, Dai X, Zheng Met al. High-resolution geochemical record for the last 1100yr from Lake Toson, northeastern Tibetan Plateau, and its climatic implications. Quaternary International, 2018, 487: 61-70.

[14] Fu X, Zhang JW, Wang L et al. Recent human impacts on sedimentary record: A case from Lake Toson. Quaternary Research, 2016, 36(6): 1456-1465. [付霞, 张家武, 王林等. 近期人类活动对湖泊沉积记录的影响一以托素湖为 例. 第四纪研究, 2016, 36(6) : 1456-1465.] 
[15] Long QF, Feng XY, Liu J et al. Microbial diversity of Keluke-Tuosu Lake wetland reserve in Qinghai-Tibet Plateau. Earth and Environment, 2017, 45(4) : 399-407. [龙启福, 封希媛, 刘静等.青藏高原克鲁克-托素湖湿地系统微生物多样 性. 地球与环境, 2017, 45(4) : 399-407.]

[16] Yang YP, Ma XY, Wang L et al. Evaluation of three methods used in carbonate content determination for lacustrine sediments. J Lake Sci , 2016, 28(4) : 917-924. DOI: 10.18307/2016.0426. [阳亚平, 马雪洋, 王林等. 湖泊沉积物碳酸 盐含量 3 种测定方法的比较. 湖泊科学, 2016, 28(4) : 917-924.]

[17] Peng Y, Xiao J, Nakamura T et al. Holocene East Asian monsoonal precipitation pattern revealed by grain-size distribution of core sediments of Daihai Lake in Inner Mongolia of north-central China. Earth and Planetary Science Letters, 2005, 233 $(3 / 4): 0-479$.

[18] Wan GJ. ${ }^{210} \mathrm{~Pb}$ Dating for recent sedimentation. Quaternary Research, 1997, 17(3) : 230-239. [万国江. 现代沉积的 ${ }^{210}$ $\mathrm{Pb}$ 计年. 第四纪研究, 1997, 17(3): 230-239.]

[19] Zhou AF, Qiang MR, Zhang JW et al. Comparison of varve chronology and ${ }^{210} \mathrm{~Pb},{ }^{137} \mathrm{Cs}$ dating from the Sugan Lake sediment. Journal of Lanzhou University: Natural Sciences, 2008, 44(6): 15-18. [ 周爱锋, 强明瑞, 张家武等. 苏干湖沉 积物纹层计年和 ${ }^{210} \mathrm{~Pb},{ }^{137} \mathrm{Cs}$ 测年对比. 兰州大学学报: 自然科学版, 2008, 44(6): 15-18.]

[20] Matisoff G. Activities and geochronology of ${ }^{137} \mathrm{Cs}$ in lake sediments resulting from sediment resuspension. Journal of Environmental Radioactivity, 2017, (167) : 222-234.

[21] Liu EF, Xue B, Yang XD et al. ${ }^{137} \mathrm{Cs}$ and ${ }^{210} \mathrm{~Pb}$ chronology for modern lake sediment: a case of Chaohu Lake and Taibai Lake. Marine Geology \& Quaternary Geology, 2009, 29(6): 89-94. [刘恩峰, 薛滨, 羊向东等. 基于 ${ }^{210} \mathrm{~Pb}$ 与 ${ }^{137} \mathrm{Cs}$ 分 布的近代沉积物定年方法——以巢湖、太白湖为例. 海洋地质与第四纪地质, 2009, 29(6) : 89-94.]

[22] Zhang F, Xue B, Yao S et al. Organic carbon burial from multi-core records in Hulun Lake, the largest lake in northern China. Quaternary International, 2018, 475: 80-90.

[23] Yin ZQ, Qin XG, Wu JS et al. Multimodal grain-size distribution characteristics and formation mechanism of lake sediment. Quaternary Research, 2008, 28(2) : 345-353. [殷志强, 秦小光, 吴金水等. 湖泊沉积物粒度多组分特征及其 成因机制研究. 第四纪研究, 2008, 28(2): 345-353.]

[24] Lei GL, Zhang HC, Zhang WX et al. Characteristics of Grain-size and Sedimentation of Shell Bar Section in Salt Lake Qarhan, Qaidam Basin. Acta Sedimentologica Sinica, 2007, 25(2): 274-282. [雷国良, 张虎才, 张文翔等. 柴达木盆地察 尔汗古湖贝壳堤剖面粒度特征及其沉积环境. 沉积学报, 2007, 25(2) : 274-282.]

[25] Ju J, Zhu L, Feng J et al. Hydrodynamic process of Tibetan Plateau lake revealed by grain size: Case study of Pumayum Co. Chinese Science Bulletin, 2012, 57(19) : 2433-2441.

[26] Liu X, Vandenberghe J, An Z et al. Grain size of Lake Qinghai sediments: Implications for riverine input and Holocene monsoon variability. Palaeogeography, Palaeoclimatology, Palaeoecology, 2016, 449: 41-51.

[27] Zhu LP, Wang JB, Lin X et al. Environmental changes reflected by core sediments since 8.4 ka in Namco, Central Tibet of China. Quaternary Research, 2007, 27(4) : 588-597. [ 朱立平, 王君波, 林晓等. 西藏纳木错深水湖芯反映的 8.4 ka 以来气候环境变化. 第四纪研究, 2007, 27(4) : 588-597.]

[28] An FY, Ma HZ, Wei HC et al. Distinguishing aeolian signature from lacustrine sediments of the Qaidam Basin in northeastern Qinghai-Tibetan Plateau and its palaeoclimatic implications. Aeolian Research, 2012, 4: 17-30.

[29] Qiang M, Liu Y, Jin Y et al. Holocene record of eolian activity from Genggahai Lake, northeastern Qinghai- Tibetan Plateau, China. Geophysical Research Letters, 2014, 41(2) : 589-595.

[30] An CB, Zhao J, Tao S et al. Dust variation recorded by lacustrine sediments from arid Central Asia since $\sim 15$ cal ka BP and its implication for atmospheric circulation. Quaternary Research, 2011, 75(3) : 566-573.

[31] Zhang X, Zhou A, Zhang C et al. High-resolution records of climate change in arid eastern central Asia during MIS 3 (51600-25300 cal a BP) from Wulungu Lake, north-western China. Journal of Quaternary Science, 2016, 31 (6) : $577-586$.

[32] Dietze E, Maussion F, Ahlborn M et al. Sediment transport processes across the Tibetan Plateau inferred from robust grainsize end members in lake sediments. Climate of the Past, 2014, 10(1) : 91-106.

[33] Wu D, Chen X, Lv F et al. Decoupled early Holocene summer temperature and monsoon precipitation in southwest China. Quaternary Science Reviews, 2018, 193: 54-67.

[34] Chen D, Ma XY, Zhang YZ et al. Environmental changes during the past 400 years documented by a short core from Lake 
Gahai, eastern Qaidam Basin. J Lake Sci, 2015, 27(4) : 735-744. DOI: 10.18307/2015.0423. [陈豆, 马雪洋, 张玉枝 等. 柴达木盆地东部尔海短钻岩芯记录的过去近 400a 区域环境变化. 湖泊科学, 2015, 27(4) : 735-744.]

[35] Song L, Qiang M, Lang L et al. Changes in palaeoproductivity of Genggahai Lake over the past 16 ka in the Gonghe Basin, northeastern Qinghai-Tibetan Plateau. Chinese Science Bulletin, 2012, 57(20) : 2595-2605.

[36] Sun Q, Zhou J, Shen J et al. Environmental characteristics of Mid-Holocene recorded by lacustrine sediments from Lake Daihai, north environment sensitive zone, China. Science in China: Series D: Earth Sciences, 2006, 49(9): 968-981.

[37] Chen Z, Ma HZ, Cao GC et al. Carbonate content in the core DG03 of Lake Gahai and its paleoenvironmental significance. Journal of Salt Lake Research, 2007, 15(2): 6-11. [ 陈忠, 马海州, 曹广超等. 尔海湖 DG03 孔碳酸盐含量及其环境 意义. 盐湖研究, 2007, 15(2): 6-11.]

[38] Li SJ, Qu RK, Zhu ZY et al. A Carbonate content record of late quaternary climate and environment changes from lacustrine core TS95 in Tianshuihai Lake Basin, northwestern Qinghai-Xizang (Tibet) plateau. J Lake Sci, 1998, 10(2) : 5865. DOI: 10.18307/1998.0210. [李世杰, 区荣康, 朱照宇等. 24 万年来西昆仑山甜水海湖岩芯碳酸盐含量变化与 气候环境演化. 湖泊科学, $1998,10(2): 58-65$.

[39] Zhu ZJ, Chen JA. Distinguishing the source of carbonates from lake sediments of Lake Chenghai, Yunnan Province. J Lake $S c i, 2009,21$ (3) : 78-82. DOI: 10.18307/2009.0311. [ 朱正杰, 陈敬安. 云南程海沉积物碳酸盐来源辨识. 湖泊科 学, 2009, 21(3): 78-82.]

[40] Huang DK, Li XQ, Jiang W et al. Geographic variation of carbonate content and pH in surface soil in East Central Asia: Significance as climate proxies. Geochimica, 2008, 37(2) : 129-138. [黄代宽, 李心清, 江伟等. 中东亚地区表土碳酸 盐含量和 $\mathrm{pH}$ 值的空间变化及其气候代用指标意义. 地球化学, 2008, 37(2)：129-138.]

[41] Zhao JJ, Lv YB, An CB et al. Carbonates in lacustrine sediments of Lake Balikun, Xingjiang, China. Marine Geology \& Quaternary Geology, 2010, 30(6) : 125-131. [ 赵家驹, 吕雁斌, 安成邦等. 新疆巴里坤湖沉积物的碳酸盐分析. 海 洋地质与第四纪地质, 2010, 30(6) : 125-131.]

[42] Zhang EL, Shen J, Wang SM et al. Climate and Environment Change during the Past 900 Years in Qinghai Lake. J Lake $S c i, 2002,14(1)$ : 32-37. DOI: 10.18307/2002.0105. [张恩楼, 沈吉, 王苏民等. 青海湖近 900 年来气候环境演化 的湖泊沉积记录. 湖泊科学, 2002, 14(1): 32-37.]

[43] Shen J, Zhang EL, Xia WL. Records from lake sediments of the Qinghai Lake to mirror climatic and environmental changes of the past about 1000 Years. Quaternary Research, 2001, 21(6): 508-513. [沈吉, 张恩楼, 夏威岗. 青海湖近千年 来气候环境变化的湖泊沉积记录. 第四纪研究, 2001, 21(6) : 508-513.]

[44] Meyers PA, Elisabeth Lallier-vergés. Lacustrine sedimentary organic matter records of late quaternary paleoclimates. Journal of Paleolimnology, 1999, 21(3) : 345-372.

[45] Yang B, Qin C, Wang J et al. A 3,500-year tree-ring record of annual precipitation on the northeastern Tibetan Plateau. Proceedings of the National Academy of Sciences, 2014, 111 (8) : 2903-2908. 